\title{
Subgrid Scale Modeling in Large-Eddy Simulation of Turbulent Combustion Using Premixed Flamelet Chemistry
}

\author{
A. W. Vreman • J. A. van Oijen • \\ L. P. H. de Goey • R. J. M. Bastiaans
}

Received: 20 December 2007 / Accepted: 1 May 2008 / Published online: 6 June 2008

(C) The Author(s) 2008

\begin{abstract}
Large-eddy simulation (LES) of turbulent combustion with premixed flamelets is investigated in this paper. The approach solves the filtered NavierStokes equations supplemented with two transport equations, one for the mixture fraction and another for a progress variable. The LES premixed flamelet approach is tested for two flows: a premixed preheated Bunsen flame and a partially premixed diffusion flame (Sandia Flame D). In the first case, we compare the LES with a direct numerical simulation (DNS). Four non-trivial models for the chemical source term are considered for the Bunsen flame: the standard presumed beta-pdf model, and three new propositions (simpler than the beta-pdf model): the filtered flamelet model, the shift-filter model and the shift-inversion model. A priori and a posteriori tests are performed for these subgrid reaction models. In the present preheated Bunsen flame, the filtered flamelet model gives the best results in a priori tests. The LES tests for the Bunsen flame are limited to a case in which the filter width is only slightly larger than the flame thickness. According to the a posteriori tests the three models (beta-pdf, filtered flamelet and shift-inversion) show more or less the
\end{abstract}

Paper presented on the Eccomas Thematic Conference Computational Combustion 2007, submitted for a special issue of Flow, Turbulence and Combustion.

\footnotetext{
A. W. Vreman · J. A. van Oijen · L. P. H. de Goey · R. J. M. Bastiaans ( $\varangle)$ Combustion Technology, Department of Mechanical Engineering, Technische Universiteit Eindhoven, Den Dolech 2, 5600 MB Eindhoven, The Netherlands e-mail: r.j.m.bastiaans@tue.nl
}

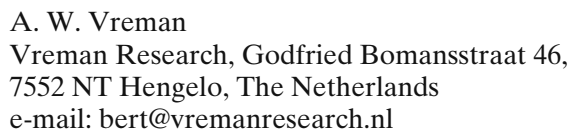


same results as the trivial model, in which subgrid reaction effects are ignored, while the shift-filter model leads to worse results. Since LES needs to resolve the large turbulent eddies, the LES filter width is bounded by a maximum. For the present Bunsen flame this means that the filter width should be of the order of the flame thickness or smaller. In this regime, the effects of subgrid reaction and subgrid flame wrinkling turn out to be quite modest. The LES-results of the second case (Sandia Flame D) are compared to experimental data. Satisfactory agreement is obtained for the main species. Comparison is made between different eddy-viscosity models for the subgrid turbulence, and the Smagorinsky eddy-viscosity is found to give worse results than eddy-viscosities that are not dominated by the mean shear.

Keywords Turbulent combustion • Premixed flamelets • Large-eddy simulation $\cdot$ Subgrid modeling

\section{Introduction}

Large-Eddy Simulation (LES) of combustion as research topic has gained an increasing amount of attention in recent years $[1,2]$. The subject is complicated, because questions regarding LES methodology and modeling issues related to chemistry need to be considered simultaneously.

In order to be able to perform three-dimensional time-dependent simulations of turbulent flows with combustion it is usually not realistic to solve transport equations for all species occurring in the chemical reaction process. Therefore, it is common to apply a reduction technique to limit the number of transport equations that need to be carried in $3 \mathrm{D}$. One group of reduction techniques is formed by the flamelet approaches (see e.g. Peters [3]). In these approaches the detailed, multidimensional chemistry is mapped to one or a few representative variables, such that the dimension of the chemistry is reduced. The mapping functions are represented by a one- or multidimensional table, constructed from a set of flamelets. Each flamelet corresponds to a one-dimensional simulation with detailed chemistry.

One specific flamelet approach is the mixture fraction/progress variable approach, which in the context of LES has been used by Pierce and Moin [4]. The mixture fraction is a conserved quantity, while the progress variable, which describes the local progress of the chemical reaction, is a suitable linear combination of appropriate chemical species. For both variables a transport equation is solved. The progress variable is a natural choice to describe the progress of the entire chemical reaction towards chemical equilibrium. The mixture fraction/progress variable approach is an efficient reduction technique, since only two additional transport equations need to be solved, while in principle, without much additional cost, all chemical species can be retrieved from the flamelet table. When the chemistry represented by the table is a good model, and the simulation is able to predict both mixture fraction and progress variable accurately, proper predictions of all chemical species can be expected.

Whereas Pierce and Moin used steady non-premixed flamelets, our mixture fraction/progress variable approach is based on steady premixed flamelets. A large part of the reaction domain cannot be represented by steady non-premixed flamelets, 
unless at the end points of a flamelet non-vanishing fluxes are imposed. Steady premixed flamelets do not have this problem; they are able to represent the entire reaction domain in a natural way [5].

To test the premixed flamelet approach [6-8] for LES, we choose a preheated premixed Bunsen flame first. The flame is similar to the flames studied by Filatyev et al. [9], Bell et al. [10], and Sankaran et al. [11], with parameters somewhat altered in our case, to enable direct numerical simulation (DNS) with moderate computational effort. Both LES and DNS in the present paper assume flamelet chemistry, but unlike LES, the DNS resolves both flame thickness and turbulence down to the Kolmogorov length-scale. Thus the DNS can be used to test and develop LES models. In particular we will consider the theory and practice of subgrid modeling needed for the quantities retrieved from the chemical flamelet database. These subgrid effects are usually treated with a presumed beta-pdf scalar approach. We will reconsider this approach in the context of LES, and also propose three alternatives: a filtered flamelet model, a shift-filter model and a shift-inversion model. The models will be compared with the presumed beta-pdf model in a priori and a posteriori tests of the premixed Bunsen flame.

The LES filter width should be smaller than the largest turbulence length-scale, since the latter needs to be resolved in LES. The implication of this constraint is that LES tests based on the DNS of the present Bunsen flame make sense only when the filter width is as large as or smaller than the flame thickness. This does not necessarily mean that the LES becomes almost as expensive as the DNS; compared to the present DNS, LES reduces the cost with more than three orders of magnitude, which is substantial. It is remarked that the Karlovitz number of the present Bunsen flame is still below the upper limit of the thin reaction zone, such that the flamelet approximation is likely to be justified [11]. LES tests for a filter width significantly larger than the flame thickness are considered in a follow-up paper [42].

In addition of validating the LES premixed flamelet approach against DNS, we wish to validate our approach against an experimental case. For this purpose we select the partially premixed diffusion flame, Sandia Flame D. For this flame a large amount of experimental information is available $[12,13]$. The flame has also been simulated many times, both LES [14-21] and RANS [22-27].

The flame is more non-premixed than premixed, and therefore it has been solved with non-premixed flamelets in the past [14]. However, the flame is not entirely non-premixed, and also highly turbulent, and because of the mixing behavior of turbulence, the use of a premixed approach is not necessarily illegitimate. Besides, as indicated above, premixed flamelets are able to fill the entire reaction regime in a natural way. Also, to know the practical range of applicability of our approach, we are interested to see how our premixed approach performs in LES for a primarily non-premixed application. Premixed flamelets have been applied to non-premixed applications before [22, 28, 29], but not in LES, as far as we know.

The outline of the paper is as follows. In Section 2 we specify the governing equations and the numerical discretization. In Section 3 we simulate the premixed Bunsen flame, with DNS and LES, where we focus on modeling the subgrid reaction effects, by comparing the four models mentioned above. In Section 4, LES-results of Sandia Flame D are compared with experimental data, while conclusions are summarized in Section 5. 


\section{Governing Equations and Discretizations}

\subsection{The unfiltered equations}

The Navier-Stokes equations with parameterized chemistry read:

$$
\begin{aligned}
\frac{\partial \rho}{\partial t}+\frac{\partial \rho u_{j}}{\partial x_{j}} & =0 \\
\frac{\partial \rho u_{i}}{\partial t}+\frac{\partial \rho u_{i} u_{j}}{\partial x_{j}} & =-\frac{\partial p}{\partial x_{i}}+2 \frac{\partial \mu S_{i j}}{\partial x_{j}}, \\
\frac{\partial \rho Z}{\partial t}+\frac{\partial \rho u_{j} Z}{\partial x_{j}} & =\frac{\partial}{\partial x_{j}}\left(\rho D \frac{\partial Z}{\partial x_{j}}\right), \\
\frac{\partial \rho Y}{\partial t}+\frac{\partial \rho u_{j} Y}{\partial x_{j}} & =\frac{\partial}{\partial x_{j}}\left(\rho D \frac{\partial Y}{\partial x_{j}}\right)+\omega_{Y}, \\
\rho & =f_{1}(Z, Y) \\
T & =f_{2}(Z, Y), \\
\omega_{Y} & =f_{3}(Z, Y),
\end{aligned}
$$

where the summation convention over repeated indices is used, while $\rho, \mathbf{u}, p, T$, $Z$ and $Y$ represent density, velocity vector, pressure, temperature, mixture fraction, progress variable, respectively.

The functions $f_{j}$ are used to define the quantities that are retrieved from a flamelet database. Since all quantities in the flamelet database can uniquely be tabulated as function of $Z$ and $Y$, the entire chemistry depends two variables only, $Z$ and $Y$. The flamelet database is a set of flamelets, obtained by solving the premixed flamelet equations with detailed chemistry using the GRI 3.0 reaction scheme [5].

The progress variable should be chosen such that its behavior on the flamelet is monotonic. The progress variable used in the partially premixed combustion case is a (linear) combination of species produced by the combustion of methane

$$
Y=Y_{\mathrm{H}_{2}} / M_{\mathrm{H}_{2}}+Y_{\mathrm{H}_{2} \mathrm{O}} / M_{\mathrm{H}_{2} \mathrm{O}}+Y_{\mathrm{CO}_{2}} / M_{\mathrm{CO}_{2}},
$$

where $Y_{i}$ and $M_{i}$ represent the mass fraction and the molar mass of species $i$, respectively. For the premixed Bunsen flame, we use another progress variable, the mass fraction of $\mathrm{O}_{2}$. In a fully premixed case, the mixture fraction is constant, such that then the equation for $Z$ can be abandoned. For constant $Z$ it is convenient to use a scaled progress variable, $c$, varying between zero (unburned) and one (burned). Thus in our premixed case, (3) and (4) are replaced by the equation for $c$ :

$$
\frac{\partial \rho c}{\partial t}+\frac{\partial \rho u_{j} c}{\partial x_{j}}=\frac{\partial}{\partial x_{j}}\left(\rho D_{c} \frac{\partial c}{\partial x_{j}}\right)+\omega_{c},
$$

with $\omega_{c}=\omega_{Y}$. Then the functions $f_{1}$ to $f_{3}$ depend on $c$ only. 
It is remarked that $T$ is almost reversely proportional to $\rho ; T$ is prescribed by the combustion approximation, an approximate equation of state [5]. In addition the rate of strain is defined by

$$
S_{i j}=\frac{1}{2}\left(\frac{\partial u_{i}}{\partial x_{j}}+\frac{\partial u_{j}}{\partial x_{i}}-\frac{2}{3} \frac{\partial u_{k}}{\partial x_{k}} \delta_{i j}\right),
$$

while the viscosity $\mu$ is a function of temperature according to Sutherland's threecoefficient law. The diff usivity $\rho D$ is the diffusivity of temperature,

$$
\lambda / c_{p}=2.58 \cdot 10^{-5}(T / 298 \mathrm{~K})^{0.69},
$$

where $\lambda$ is the thermal conductivity and $c_{p}$ the specific heat [30]. The diffusivity $D_{c}$ in the premixed case is equal to $D$ divided by the Lewis number of $\mathrm{O}_{2}(1.106)$. In the partially premixed case (flame D) we use $D_{Z}=D_{Y}=D$.

\subsection{The filtered equations}

Whereas DNS with flamelet chemistry solves the unfiltered equations, LES solves the filtered equations. The basic filter notation in LES reads:

$$
\overline{\rho u_{i}}=\int_{\Omega} G(\mathbf{x}, \xi) \rho u_{i} d \xi
$$

where $\Omega$ is the flow domain and $G$ is the filter kernel. Implementations in the present work use the top-hat filter, defined by

$$
G(x, \xi)=1 / \Delta^{3} \quad \text { if } \quad\left|x_{i}-\xi_{i}\right|<\Delta_{i}, \quad i=1,2,3 ;
$$

and zero otherwise. Here $\Delta$ denotes the filter width, equal to $\left(\Delta_{1} \Delta_{2} \Delta_{3}\right)^{\frac{1}{3}}$, and we use $\Delta_{i}=h_{i}$, where $h_{i}$ is the local mesh-spacing in the $x_{i}$-direction. In variable density flows it is convenient to use the density-weighted or Favre filter as well, defined by $\tilde{u}_{i}=\overline{\rho u}_{i} / \bar{\rho}$.

The filtered equations are obtained by application of the Favre average to the equations in the previous subsection. The nonlinearities in the equations lead to unknown terms, which are either modeled, or neglected. There are four types of subgrid terms: subgrid terms arising from the nonlinearity of the convective terms, subgrid terms arising from the nonlinearity of the chemical parametrization (nonlinearities in $f_{1}$ to $f_{3}$ ), subgrid terms arising from the nonlinearity of the viscous terms, and subgrid terms due to the lack of commutation of filter and derivative on nonuniform grids. Types three and four are neglected in the present work, type two will be considered in detail in Section 3, and for type 1 the eddy-viscosity/eddydiffusivity closure approach is adopted. As an example, we write out the resulting equation used for $c$ :

$$
\frac{\partial \bar{\rho} \tilde{c}}{\partial t}+\frac{\partial \bar{\rho} \tilde{u}_{j} \tilde{c}}{\partial x_{j}}=\frac{\partial}{\partial x_{j}}\left(\left(\bar{\rho} D_{Y}+\frac{\mu_{t}}{S c_{t}}\right) \frac{\partial \tilde{c}}{\partial x_{j}}\right)+\overline{\omega_{c}},
$$


The last term is still in unclosed form (closures for this term will be presented in Section 3). The eddy-diffusivity is the ratio of the eddy-viscosity and a constant Schmidt number of 0.4 , according to literature [14]. Unless mentioned otherwise, we use for the eddy-viscosity the following model [31]:

$$
\mu_{t}=\bar{\rho} C\left(\frac{\beta_{11} \beta_{22}-\beta_{12}^{2}+\beta_{11} \beta_{33}-\beta_{13}^{2}+\beta_{22} \beta_{33}-\beta_{23}^{2}}{\alpha_{k l} \alpha_{k l}}\right)^{1 / 2} .
$$

Here the tensor $\beta$ equals the gradient model,

$$
\beta_{i j}=\Delta_{k}^{2} \alpha_{k i} \alpha_{k j}, \quad \alpha_{k i}=\frac{\partial \tilde{u}_{i}}{\partial x_{k}}
$$

and the model constant is related to the Smagorinsky constant $C=2.5 C_{S}^{2}$. In this work we take $C_{S}=0.1$. A priori tests have shown that the gradient model [32] mimics the structure of the exact turbulent stress quite accurately [33]. Since straightforward use of the gradient model causes stability problems, the gradient model was recast into the eddy-viscosity formulation above. Model (15) has shown to be as accurate as the dynamic subgrid model [34] in wall-bounded and free shear flow and, like the dynamic model and the wall-adapted local eddy-viscosity model [35], but unlike the Smagorinsky model, the present model vanishes near walls and in transitional flow. In fact it vanishes exactly for certain types of laminar flow, the same types of flow for which the exact subgrid dissipation is zero [31]. Comparison with the other eddyviscosity models just mentioned will be made in Section 4.

\subsection{Numerical discretization}

We use a straightforward and efficient implementation to solve the equations with parameterized chemistry numerically. The variable density approach involves a Poisson equation for the pressure, similar to other low-Mach methods $[4,14]$.

For the continuity and momentum equations the standard finite volume method is employed, with second-order central differencing on a staggered Cartesian mesh. The discrete convective terms would conserve kinetic energy if the density were constant. The time integration of the momentum equations is explicit, Adams-Bashforth for the convective and forward Euler for the viscous terms. This hybrid timestepping method has better linear stability properties than pure Adams-Bashforth or pure forward Euler. This is an advantage for the momentum equation, for which dissipation can be low, since central differencing is used for the spatial derivatives. Because of its low numerical dissipation, central differencing is in general preferred over upwind schemes in direct and large-eddy simulations of turbulent flows.

The three scalar equations are recast into the equivalent advective formulations. Then the Van Leer's third-order accurate MUSCL scheme [36], which is TVD, is applied to the advective terms. Thus the spatial discretization of the scalar equations introduces numerical diffusion, which is not the case in the momentum equations. However, for the scalar equations numerical diffusion is hard to avoid if we want to keep the scalars in between their physical bounds. We use explicit Euler for both advective and diffusive terms in the scalar equations, since the MUSCL scheme introduces sufficient dissipation to remain sufficiently far away from the imaginary axis in the stability regime. 
The algorithm that is used to update the variables from level $n$ to $n+1$ consists of five steps.

The first step is to obtain the scalars at level $n+1$. Since the spatial discretization of the scalars is based on the advective formulation, $\rho$ at level $n+1$ is not required to obtain the scalars at level $n+1$.

In the second step we compute the viscous minus convective terms in the momentum equation, their sum is denoted by $\mathbf{q}$,

$$
q_{i}=\left(\frac{\partial 2 \mu S_{i j}}{\partial x_{j}}\right)_{n}-\frac{3}{2}\left(\frac{\partial \rho u_{i} u_{j}}{\partial x_{j}}\right)_{n}+\frac{1}{2}\left(\frac{\partial \rho u_{i} u_{j}}{\partial x_{j}}\right)_{n-1},
$$

and we obtain the uncorrected momentum by

$$
\mathbf{w}=\hat{\rho}^{n} \mathbf{u}^{n}+\delta t \mathbf{q}
$$

where $\delta t$ is the time-step, and $\hat{\rho}$, the average of the density of the two nearest neighboring points, needed to obtain the density at the staggered velocity location in between. The convective momentum fluxes on the faces of the staggered cells are computed as the products of velocities multiplied with the density on the faces. However, the density is defined at the pressure cell centers (like the scalars). Averages involving the smallest possible number of density cells are used to obtain the density on the faces of the velocity cells. For example, if we compute the momentum flux through the $x_{1}$ face of the $u_{1}$ cell, the density is available right there; no density interpolation is required. But if we compute the momentum flux through $x_{2}$ or $x_{3}$ faces of the $u_{1}$ cell the density needs to be interpolated from four points corresponding to the corners of a surrounding square.

The third step is to calculate $\rho^{n+1}, T^{n+1}$ and $w_{c}^{n+1}$ from the flamelet database where the scalars at level $n+1$ serve as entries (using linear interpolation of the values in the table).

In the fourth step a Poisson equation is solved to obtain the pressure. To obtain the Poisson equation, the unknown momentum at level $n+1$,

$$
\hat{\rho}^{n+1} \mathbf{u}^{n+1}=\mathbf{w}-\nabla(p \delta t),
$$

is substituted into the continuity equation

$$
\frac{\partial \hat{\rho}^{n+1} u_{i}^{n+1}}{\partial x_{i}}=-\frac{\rho^{n+1}-\rho^{n}}{\delta t} .
$$

Thus the Poisson equation reads

$$
\frac{\partial^{2}(p \delta t)}{\partial x_{i}^{2}}=\frac{\rho^{n+1}-\rho^{n}}{\delta t}+\frac{\partial w_{i}}{\partial x_{i}} .
$$

It is solved by a multigrid method using $V$-cycles and the SOR smoother (lexicographical Gauss-Seidel with overrelaxation factor 1.5). The restriction operator is the uniform average from eight small cells to a large cell, while trilinear interpolation [37] is used as prolongation operator.

The fifth and last step provides the momentum at the new time level by evaluating (19) from which the velocity at the new time level directly follows.

In contrast to what is sometimes believed, the use of a first-order time discretization in the continuity equation does not produce an inconsistent (zeroth order) 
numerical scheme. Substitution of (18) into (21) and subsequent substitution of (20) evaluated at levels $n$ and $n+1$ show that the Poisson equation is equivalent to

$$
\frac{\partial^{2} p}{\partial x_{i}^{2}}=\frac{\rho^{n+1}-2 \rho^{n}+\rho^{n-1}}{(\delta t)^{2}}+\frac{\partial}{\partial x_{i}}\left[-\frac{\partial \rho u_{i} u_{j}}{\partial x_{j}}+2 \frac{\partial \mu S_{i j}}{\partial x_{j}}\right] .
$$

In the first term at the right-hand side we recognize the discretization of a secondorder time derivative. In fact (22) is the acoustic wave equation, that is enforced when we solve (21). If we define the pressure at level $n,(22)$ represents a Poisson equation that is second-order accurate in time. However, defining the pressure at step $n$, causes the velocity correction (step six) to be first-order accurate in time. Thus the global treatment of pressure is first-order accurate, which is consistent, nevertheless.

Implicit or semi-implicit methods are often used to increase robustness. Robustness for variable-density flows is harder to achieve than for incompressible flow. The reason is that the velocity divergence is non-zero in variable-density flows. As a result the total kinetic energy is not necessarily conserved in the absence of viscous forces, because the non-conservative pressure velocity divergence term does not vanish. In particular, stability is a problem for large density ratios in combination with high Reynolds number. In such cases we propose to smooth the density with a spatial filter of width $2 h$, directly after the density is retrieved from the flamelet database. More specifically, a three-points filter with weights $\frac{1}{4}, \frac{1}{2}, \frac{1}{4}$ is applied to the density three times, one time in each direction. This is a second-order accurate operation, such that the order of accuracy of the numerical scheme is not affected. For the large-eddy simulations of flame D, reported in Section 4, the $2 \mathrm{~h}$-filter was found to enhance robustness significantly (see Ref. [21], for an illustration of several effects of the density filter). However, the density filter was not applied in the direct and large-eddy simulations of the preheated premixed Bunsen flame (Section 3), since Reynolds number and density ratio in that flow are small compared to flame D.

\section{DNS and LES of a Preheated Premixed Bunsen Flame}

In this section we consider a planar Bunsen flame. In the first subsection we define the flow and present a DNS that was performed with flamelet chemistry. Then we consider four models for the subgrid chemistry in LES (Section 3.2). A priori tests of these models are presented in the third subsection, and a posteriori tests in the fourth.

\subsection{Direct numerical simulation}

The configuration of the planar methane-air Bunsen flame is a spatial jet of the unburnt mixture (mean centerline velocity $30 \mathrm{~m} / \mathrm{s}$ and $T=800 \mathrm{~K}$ ), surrounded by a co-flow with hot products (velocity $7.5 \mathrm{~m} / \mathrm{s}$ and $T=2,204 \mathrm{~K}$ ). The slot width of the burner equals $2.4 \mathrm{~mm}$. The preheated flame is premixed with equivalence ratio 0.7 . Some premixed jet flames in industry also operate at a high temperature. The simulation has been inspired by the DNS performed by Sankaran et al. [11], who simulated a similar flame with detailed chemistry, using 5,000 processors. To 
diminish the computational demand of the DNS significantly, we choose to model the chemistry with a premixed flamelet. To remain within the regime of validity of the flamelet approach, the ratio of Kolmogorov scale and flame thickness needs to be sufficiently large. To increase the Kolmogorov scale we increase the domain size and decrease the velocity both with a factor two compared to Sankaran et al. Another difference are the inflow conditions. Whereas Sankaran et al. used a separate temporal simulation to prescribe the time-dependent inflow, we perturb the central jet with random uniform noise, which is filtered to control the turbulence length-scale. In some experiments [9] the inflow turbulence is generated by a suitable grid to set the length-scale of the turbulence.

The size of the simulation domain is $7.2 \times 7.2 \times 14.4 \mathrm{~mm}$. The $z$-direction is streamwise, the $y$-direction is across the jet, whereas the $x$-direction is spanwise and homogeneous. Periodic boundary conditions are used in the spanwise directions. The outflow boundary conditions in the normal and streamwise direction assume Neumann conditions for the three velocity components. The pressure satisfies Neumann conditions at the streamwise in- and outflow, while it is held constant in the normal outflow planes. Note that although the density is varying, the algorithm is essentially incompressible (the pressure is solved with a Poisson equation). Therefore the convective outflow conditions, commonly used in compressible flow solvers, are not mandatory. The present incompressible boundary conditions are very similar to zero stress boundary conditions, that are suitable outflow conditions for incompressible flow (Wesseling [37], p. 246).

The inflow is prescribed by hyperbolic tangent functions for the streamwise velocity and progress variable $(c)$. These functions assume their maximum slope at $|y|=1.2 \mathrm{~mm}$, while the thickness of the profiles based on maximum slope is set to $0.35 \mathrm{~mm}$, approximately equal to the thickness of $c$ obtained from the premixed flamelet. Thus, in the inflow plane $c$ is zero for $|y| \ll 1.2 \mathrm{~mm}, 0.5$ at $|y|=1.2 \mathrm{~mm}$, and one for $|y| \gg 1.2 \mathrm{~mm}$.

The progress variable enters without perturbation, but the three velocity components enter with a large turbulence intensity of $6.9 \mathrm{~m} / \mathrm{s}$ each. The inflow velocity perturbations for the Bunsen flame are constructed such that they are the same on each grid used. The inflow conditions are filtered such that the inflow condition of the DNS does not contain wave numbers that are subgrid with respect to the LESgrid later on. In this way contributions that are subgrid with respect to the LES-grid do not enter by the inflow condition; the subgrid scales are generated by large scales during the evolution of the flow.

In more detail, for each velocity component, and each $\delta t_{0}=5 \cdot 10^{-8}$, random numbers between -1 and 1 are generated on a $384^{2}$ mesh (with the same length as the inflow plane). Then these random numbers are filtered, applying a box-filter of $l_{0}=1.2 \mathrm{~mm}$ in the spatial directions, and a temporal exponential filter. For a signal $f$, the temporal filter is discretized by

$$
\hat{f}_{n+1}=\left(1-\frac{\delta t_{0}}{l_{0} / U_{0}}\right) \hat{f}_{n}+\frac{\delta t_{0}}{l_{0} / U_{0}} f_{n} .
$$

The perturbation is initialized with $\hat{f}_{0}=0$. The spatial inflow filter requires boundary conditions to be specified after is applied; periodic boundary conditions are imposed in both directions. After the filtering, the perturbation is confined to the center jet, 
Fig. 1 Two-point autocorrelation functions of streamwise (solid), normal (dashed) and spanwise (dotted) velocity fluctuation as a function of distance $r$ in the spanwise direction, for the streamwise locations $z=0$ (symbols) and $z=3.6 \mathrm{~mm}$ (no symbols). The autocorrelation functions were obtained from a snapshot of the DNS-field and integrated across the normal direction

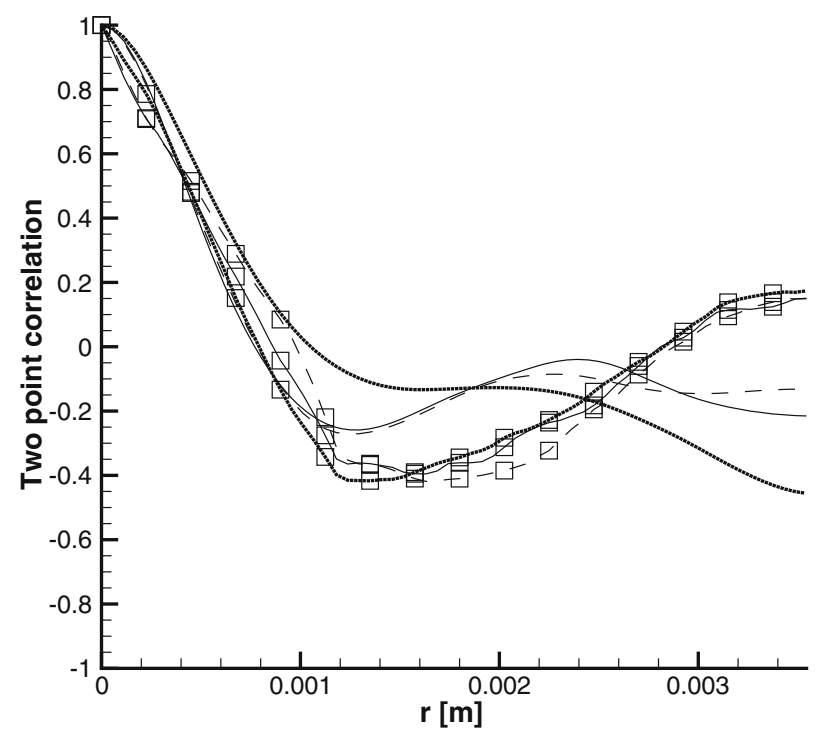

using a tangent hyperbolic function of the same shape as the mean inflow profiles. Subsequently, the perturbation is interpolated to the simulation grid, multiplied by 3,000 to obtain the level the inflow turbulent intensities mentioned, and finally the perturbation is superimposed on the mean inflow profiles.

The time scale of the inflow perturbation $\delta t_{0}$ is such that after multiplication with $U_{0}$ the width of the temporal filter equals the spatial length $l_{0}$ (width definitions are based on the second moments of the filters). In the experiments a physical grid is sometimes used to generate and control turbulence inside the nozzle. The length scale $l_{0}$ represents the size of that grid. In the present case $l_{0}$ has been chosen such that the turbulent structures are large enough to persist downstream and small enough to fit into the computational box. According to Fig. 1, the fall-off of the three two-point autocorrelation functions is reasonable, albeit not sufficient to eliminate all effects of the spanwise periodic boundary condition. To limit computation time, a trade-off has to be made between sufficient resolution of smallest length-scales and sufficient distance between periodic boundary conditions. The former issue is mandatory to obtain numerically reliable computations, while the latter issue is desirable to obtain a more realistic flame.

The basic DNS (A) in this section is performed with a time-step of $2 \cdot 10^{-7} \mathrm{~s}$ on grid A, which contains $128 \times 128 \times 256$ cells $\left(\delta t_{0}\right.$ was chosen four times smaller such that if temporal refinement were needed, this would not alter the inflow perturbation). To verify this DNS, a coarser DNS (B) is performed, with a time-step of $4 \cdot 10^{-7} \mathrm{~S}$ and a grid that is coarsened with a factor two in each direction. In both cases the grid size is uniform, $0.056 \mathrm{~mm}$ in DNS A and $0.11 \mathrm{~mm}$ in DNS B. Statistical averaging starts at $t=0.0006$, while the simulations are stopped at $0.004 \mathrm{~s}$, which corresponds to approximately 8 flow-through times.

A snapshot of $c$ obtained from a vertical plane of DNS A is shown in Fig. 2. Also a laminar flame simulation is shown, using the same grid size in a little larger 
Fig. 2 Contours of progress variable in the vertical plane $x_{1}=3.6 \mathrm{~mm}$, for the turbulent case (a DNS A; instantaneous; $t=0.004 \mathrm{~s})$ and for the laminar case (b)

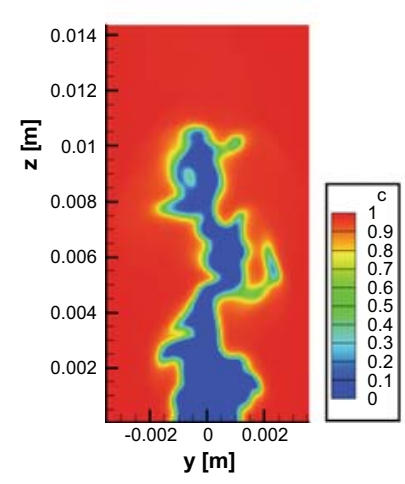

(a)

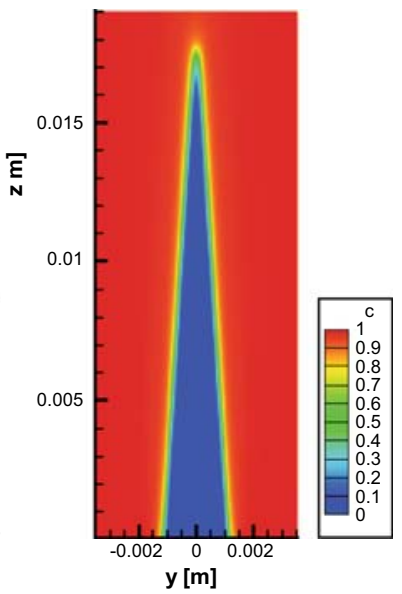

(b)

two-dimensional domain. The laminar flame is much taller than the turbulent flame. The burning velocity, or mass consumption rate, is defined by

$$
s=\frac{m}{\rho_{u} A},
$$

where $A$ represents the mean surface of the flame (here the isosurface $\bar{c}=0.6), m$ the mass flow through that surface, and $\rho_{u}$ the density of the unburned mixture. Because of the conservation of mass, the value of $m$ is equal to the mass flux through the surface enclosed by the contour $\bar{c}=0.6$ on the inflow plane. The flamelet burning velocity $S_{L 0}$ equals $1.77 \mathrm{~m} / \mathrm{s}$. The burning velocity of the laminar simulation is very close, $1.74 \mathrm{~m} / \mathrm{s}$. The burning velocity is fairly independent of the level of the isosurface; for the isosurface 0.5 instead of 0.6 , we obtain $1.73 \mathrm{~m} / \mathrm{s}$. The laminar burning velocity for a coarser mesh (grid size doubled) equals $1.72 \mathrm{~m} / \mathrm{s}$, from which we conclude that the laminar burning velocity is almost grid independent. The turbulent burning velocity $\left(s_{T}\right)$ is much higher, $3.73 \mathrm{~m} / \mathrm{s}$ for DNS A, and $3.71 \mathrm{~m} / \mathrm{s}$ for DNS B. The ratio $s_{T} / s_{L 0}$ is 2.1 , not far from the range that was measured experimentally for turbulent planar (non-preheated) Bunsen flames [9].

Figure 3a shows the turbulence intensities for both resolutions. They appear to be well-resolved by the coarse-grid DNS already. Also the flame thickness is properly resolved, since also the fluctuations of the chemical source term are grid independent (Fig. 3b).

Three turbulence length-scales based on the turbulent dissipation rate $\epsilon$ are shown in Fig. 4, the length-scale of large structures, $l=\left(u_{3}^{\prime}\right)^{3} / \epsilon(\epsilon$ is the turbulent dissipation rate), the Kolmogorov length-scale $\eta=\left(v^{3} / \epsilon\right)^{1 / 4}$, and the 'streamwise' Taylor microscale $\lambda$ defined as the root of $\left.<u_{3}^{\prime} u_{3}^{\prime}\right\rangle /<\left(\partial u_{3}^{\prime} / \partial x_{3}\right)^{2}>$. For the determination of these statistics, temporal and spanwise averaging of the appropriate moments needed for this quantities is performed. The velocity derivative in the Taylor microscale is approximated with central differences, while the calculation of the turbulent dissipation reuses the strain-rates calculated for the momentum equation. The peak of $l=\left(u_{3}^{\prime}\right)^{3} / \epsilon$ near the outlet $(z>0.012 \mathrm{~m})$ is due to both an increase of $u_{3}^{\prime}$ (Fig. 3a) and a decrease of $\epsilon$ (not shown). The decrease of $\epsilon$ is probably physical, since the 


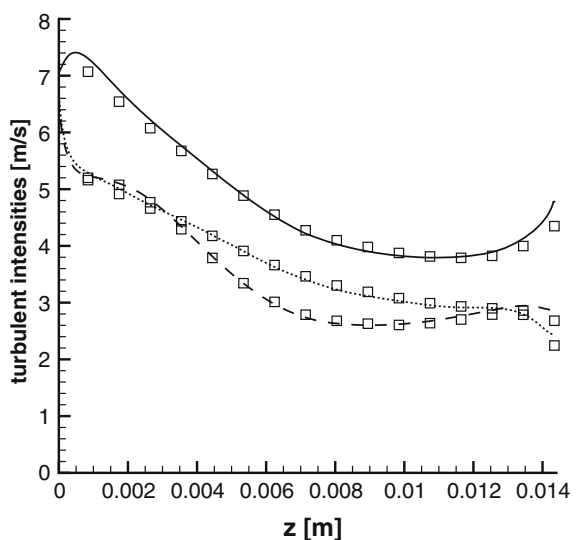

(a)

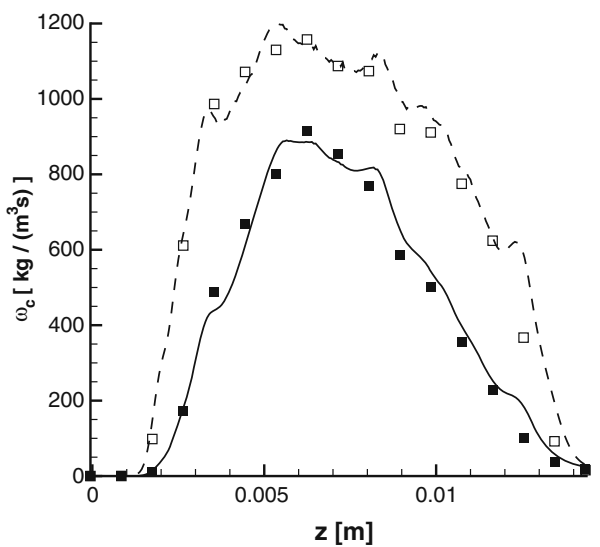

(b)

Fig. 3 Centerline results for DNS A (curves) and DNS B (symbols). a Turbulence intensities, streamwise (solid), normal (dashed) and spanwise (dotted). b Mean (solid) and rms (dashed) of chemical source term $\omega_{c}$

turbulence at the hot side of the flame is damped by the strong increase of kinematic viscosity $(\mu / \rho)$ with temperature. However, the $25 \%$ increase of $u_{3}^{\prime}$ near the outlet could be an effect of boundary condition. According to the extent of this cusp, the downstream influence of the outlet boundary extends to a modest distance of $1 \mathrm{~mm}$ from the outflow boundary condition.

Near the inlet $(z \approx 1 \mathrm{~mm})$, the turbulence corresponds to $R e_{\lambda} \approx 45$. Using the flamelet thickness based on the maximum temperature gradient, $l_{F} \approx 0.3 \mathrm{~mm}$, and taking for the Kolmogorov length-scale an average value of roughly $\eta \approx 0.1 \mathrm{~mm}$, we find a Karlovitz number around 9. According to Peters [38], the turbulent flame is in the thin reaction zone.

Fig. 4 Turbulence length-scale $l$ (solid), $\lambda$ (dashed) and $\eta$ (dotted) for DNS A

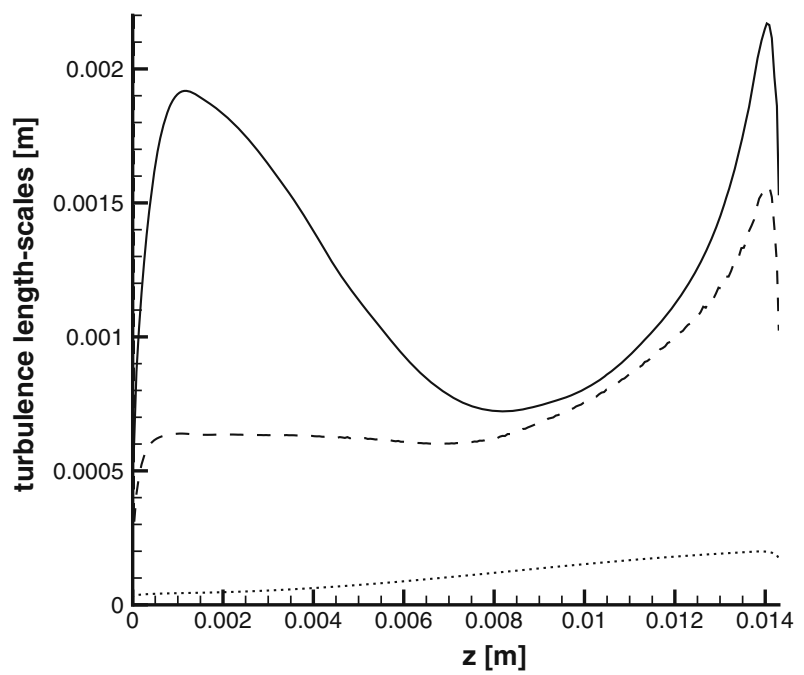




\subsection{Four SGS modeling approaches for chemical variables}

When performing LES of reactive flows, we need closures for unknown terms, such as $\overline{\omega_{c}(c)}, \overline{\rho(c)}, \widetilde{T(c)}$, and filtered mass fractions stored in the flamelet table. We consider a number of models for these unknown terms. It is sufficient to give the different formulations for $\overline{\omega_{c}(c)}$ only, since in each approach the modeling of the other unknowns just mentioned is completely analogous to the modeling of $\overline{\omega_{c}(c)}$. Thus the unknown filtered source term, $\overline{\omega_{c}(c)}$, is modeled by $\omega_{M i}$, referring to model $M i$ for the source term. The trivial model requires no modeling effort: $\omega_{M 0}=\omega_{c}(\tilde{c})$. We want a model to perform at least as well as M0.

The first nontrivial model that we consider is the presumed beta-pdf model. Its use for LES has been proposed by Cook and Riley [39] and since then it has become a standard closure technique in LES, like in RANS. That the beta-pdf probably provides a suitable (although non-exact) description of chemistry subgrid terms, can be seen from the following transformation of the filter integral in one-dimension:

$$
\begin{aligned}
\overline{\omega_{c}(c)} & =\int_{-\infty}^{\infty} G(x, \xi) \omega_{c}(c(\xi)) d \xi \\
& =\int_{0}^{1} G(x, \xi(c)) \omega_{c}(c)\left|\frac{d \xi}{d c}\right| d c=\int_{0}^{1} G(x, \xi(c)) \frac{1}{|d c / d \xi|} \omega_{c}(c) d c .
\end{aligned}
$$

Since we search for a pdf that has the same moments as the filter function $G$, the pdf should equal $G(x, \xi(c)) /|d c / d \xi|$. The latter function depends on the spatial derivative of $c$, and therefore, it does not equal the usual presumed beta-function exactly. However, if we consider a typical tangent hyperbolic profile and a filter width larger than the flame thickness, we see that the probability is large near 0 and near 1. This feature is covered by the beta-pdf, since the beta-pdf includes (integrable) singularities near the end-points.

In practice the beta-pdf approach is used to construct a table, where the filtered

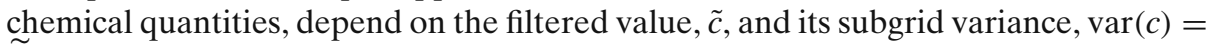
$\widetilde{c^{2}}-\tilde{c}^{2}$. The model for the source-term (M1) reads

$$
\omega_{M 1}=\int_{0}^{1} \omega_{c}(c) P_{\tilde{c}, \operatorname{var}(c)}(c) d c,
$$

where $P$ represents a probability density function of beta-shape with mean $\tilde{c}$ and variance $\operatorname{var}(c)$. While constructing the table, the beta-pdf integrals have to be calculated carefully [26] and need to be checked for their convergence. The closure problem that remains is to provide a suitable model for $\operatorname{var}(c)$. A similarity or gradient model is often adopted, e.g.,

$$
\operatorname{var}(c) \approx \frac{a^{2} \Delta_{k}^{2}}{12}\left(\frac{\partial \tilde{c}}{\partial x_{k}}\right)^{2},
$$

where $a$ is assumed to be constant, or determined by a dynamic procedure. For smooth fields on the scale of $\Delta$ we can deduce $a=1$ from a simple Taylor expansion. Next we derive an upperbound for $a$. The beta-pdf requires $\tilde{c}(1-\tilde{c}) \leq \frac{1}{4}$. Since we discretize $\nabla \tilde{c}$ in (27) with second-order central differencing across a distance of $2 \Delta$, 
the absolute maximum of a first-order derivative of $\tilde{c}$ is $1 /(2 \Delta)$. It follows that for $a$ larger than 2, values larger than the theoretical beta-pdf maximum $\left(\frac{1}{4}\right)$ may occur.

To circumvent the modeling of $\operatorname{var}(c)$, we propose a simpler and more straightforward approach than the beta-pdf. Equality (25) indicates that when the beta-pdf is evaluated, the flamelet is filtered in $c$-space. However, it is more straightforward to filter the flamelet directly in physical space. Since in the present case $\Delta$ is uniform, we need to filter the flamelet only once for a given LES grid. Thus the one-dimensional flamelet functions $\omega_{c}(x)$ and $c(x)$ are filtered to $\omega_{f}$ and $c_{f}$ respectively, in $x$-space, using the one-dimensional top-hat filter with filter width $\Delta$ to obtain $\omega_{f}(x)$, and the corresponding Favre filter to obtain $c_{f}(x)$. Subsequently $\omega_{f}$ is written as function of $c_{f}$, and this function $\omega_{f}\left(c_{f}\right)$ (the flamelet parametrization) is stored in a table. Thus, the filtered flamelet model (M2) used in the 3D simulations reads

$$
\omega_{M 2}=\omega_{f}(\tilde{c}) .
$$

When filtering the flamelet, $\Delta$ should match the units of the spatial coordinate of the flamelet. For nonuniform $\Delta$, the flamelet should be filtered for multiple values of $\Delta$, and a dimension dependent on $\Delta$ should be added to the look-up table used in LES. Filtered flamelets can also be used to construct a probability density function [40], but as in the beta-pdf approach, a model for $\operatorname{var}(c)$ is required then.

In the third model, the resolved source term is explicitly filtered

$$
\omega_{M 3}=H \omega_{c}(\tilde{c}),
$$

The explicit filter is denoted by $H$, and its filter width equals $a \Delta$ with $a \geq 1$. It is discretized using three subsequent one-dimensional three-point filters with discrete weights $a^{2} / 24$ for the two outer points and $\left(1-2 a^{2}\right) / 24$ for the central point. We call this model the shift-filter model, since, compared to the basic filter, the explicit filter 'cut-off' wavenumber is shifted towards a smaller wavenumber $(\pi /(a \Delta)$ instead of $\pi / \Delta)$.

Inverse filtering can also be used to model the reaction source terms in LES, see Geurts [41], who used both geometric series inversion and exact numerical inversion for this purpose. Since due to the limited resolution any inversion can retrieve information of the subgrid scales only partially, inversion modeling is expected to underestimate the effect of subgrid scales. This may be compensated by the use of the shift-filter. We thus propose the shift-inversion model

$$
\omega_{M 4}=H \omega_{c}\left(H^{-1} \tilde{c}\right), \quad H^{-1} \approx \tilde{c}-\frac{a^{2} \Delta_{k}^{2}}{24} \frac{\partial^{2} \tilde{c}}{\partial x_{k}^{2}},
$$

in which the oldest technique of inverse filtering has been used, the Taylor expansion in $\Delta[32,33]$. The second-order derivatives evaluated with the standard second-order central difference on three points. A disadvantage of the present filtering method is that $H^{-1} \tilde{c}$ may lie outside the physical bounds 0 or 1 . Thus the quantity is clipped to remain between 0 and 1 .

The subgrid models M0-4 used for the subgrid reaction effects, differ with respect to their ability to deal with subgrid wrinkling of the flame. It seems that only models M1 and M4 have intrinsic mechanisms to account for subgrid wrinkling; $\operatorname{var}(c)$ in M1 and $H^{-1}$ in M4 include subgrid wrinkling effects. The success of model M2 
(which will appear later on) indicates that in the present application the effect of subgrid wrinkling of the flame is marginal. This suggests that the smallest scale of flame wrinkling is larger than the smallest scale of the turbulence. In the present case the smallest scale of flame wrinkling is probably determined by the flame thickness ( $0.3 \mathrm{~mm}$ based on the maximum slope of temperature in the premixed flamelet), which is considerably larger than the Kolmogorov scale $(0.1 \mathrm{~mm})$.

\subsection{A priori tests for the chemical reaction term}

Next a priori tests for the five models described in the previous subsection are presented. For these tests, we use the instantaneous DNS field at $t=0.004 \mathrm{~s}$, but we have verified the results to be similar at another time. Test are performed for two different grids, one four times coarser than the DNS, another eight times coarser than the DNS (in each direction). Then using the top-hat filter we calculate the 'exact' $\overline{\omega_{c}(c)}$. Subsequently, the predictions of the five models formulated above are calculated, based on the filtered DNS restricted to the coarse grid. The beta-pdf model is calculated twice, once using the approximated $\operatorname{var}(c)$, and once using the 'exact' $\operatorname{var}(c)$. The latter case is labeled by M1A. It cannot be used in actual LES, but it serves to investigate which part of the error is due to the beta-pdf assumption. Figure 5 shows the spatial $L_{2}$-norms of the errors, $\left\|\omega_{M i}-\overline{\omega_{c}(c)}\right\|$ normalized by a quantity independent of filtering, $\left\|\omega_{c}(c)\right\|$. Two different LES-grids, both with $\Delta$ equal the LES-grid size have been used. Note that models M0, M1A and M2 do not depend on the parameter $a$. The lowest error is clearly provided by M1A, which indicates that the error introduced by the beta-pdf assumption is small. However, $\mathrm{M} 1 \mathrm{~A}$, is a theoretical model; in practice the beta-pdf model M1 has to be used. Unfortunately, M1 is much less accurate, which is apparently caused by the model for $\operatorname{var}(c)$. In addition, the accuracy of M1 strongly depends on the value of $a$. The optimum value is not the same for both grids, $a \approx 1.4$ for the finer and $a \approx 2$

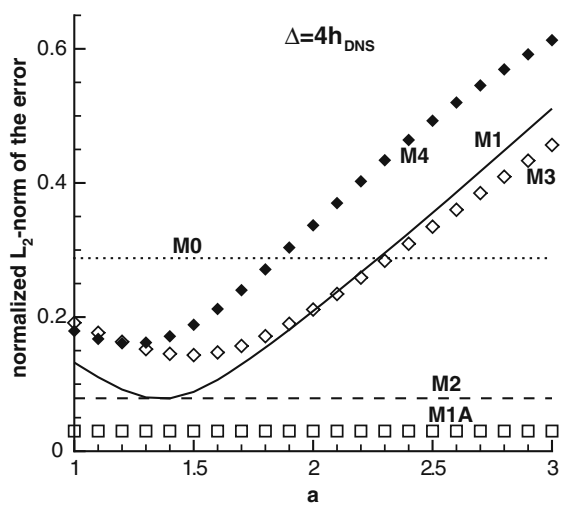

(a)

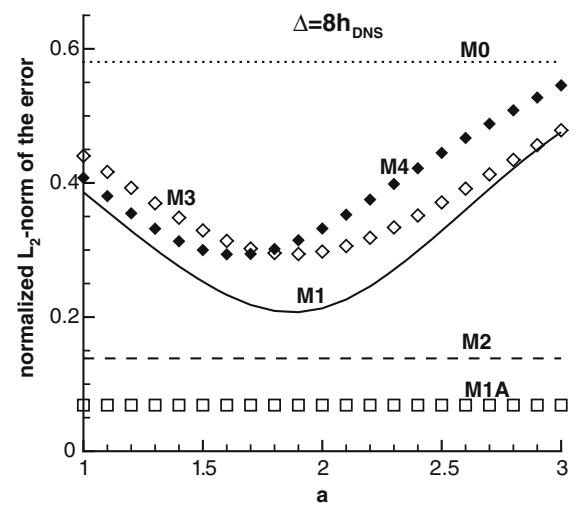

(b)

Fig. 5 Modeling errors as function of the parameter $a$. Evaluated a priori, from DNS A, on two LES-grids: $32 \times 32 \times 64(\mathbf{a})$ and $16 \times 16 \times 32(\mathbf{b})$ 
for the coarser LES-grid. This means that a dynamic model for var(c) has to be applied with care. At least the basic dynamic presumption that the model coefficient is independent of filter size seems to be invalid in the present cases. Apart from M1A, which cannot be used in actual LES, the lowest a priori error is clearly provided by the filtered flamelet model (M2).

If the presumed $\beta$-pdf approach is used, the model for $\operatorname{var}(c)$ sometimes attains larger values than the allowable maximum variance of the $\beta$-pdf distribution, which is $\tilde{c}(1-\tilde{c})$. In this case the model of the variance has to be clipped to the maximum variance. The fraction of grid points where this clipping occurs has been plotted in Fig. 6, as a function of the model parameter $a$. A result for the variance model with a dynamic coefficient has been included, showing that in $9 \%$ of the points the theoretical maximum of the $\beta$-pdf is violated. Only the points with $0.05<\tilde{c}<0.95$ were taken into account when the fractions were calculated.

\subsection{A posteriori LES compared with DNS}

Results of actual LES with different models for the reaction term are shown in Fig. 7. The size of the LES-grid is $16 \times 16 \times 32$, which corresponds to $\Delta=8 h_{D N S}$, and the time step of the LES is five times the DNS time step. The coarsening is significant, since an LES on this grid is about three orders of magnitude cheaper than the DNS. Nevertheless, the flame thickness $(0.35$ based on the maximum gradient of the progress variable in the flamelet) is not much smaller than the LES grid size $(0.45 \mathrm{~mm}))$. As the scale of wrinkling is usually not smaller than the flame thickness [38], we do not expect much subgrid wrinkling in this LES. It does not make much sense to coarsen the LES further, since then the grid becomes unsuitable for LES in the sense that the large turbulent length-scale in the DNS becomes unresolved. Thus the present comparison between LES and DNS is limited to LES which resolves

Fig. 6 Fraction of points where the model for $\operatorname{var}(c)$ predicts larger values than the $\beta$-pdf maximum variance $\tilde{c}(1-\tilde{c})$. A priori results for $\Delta=8 h_{D N S}$

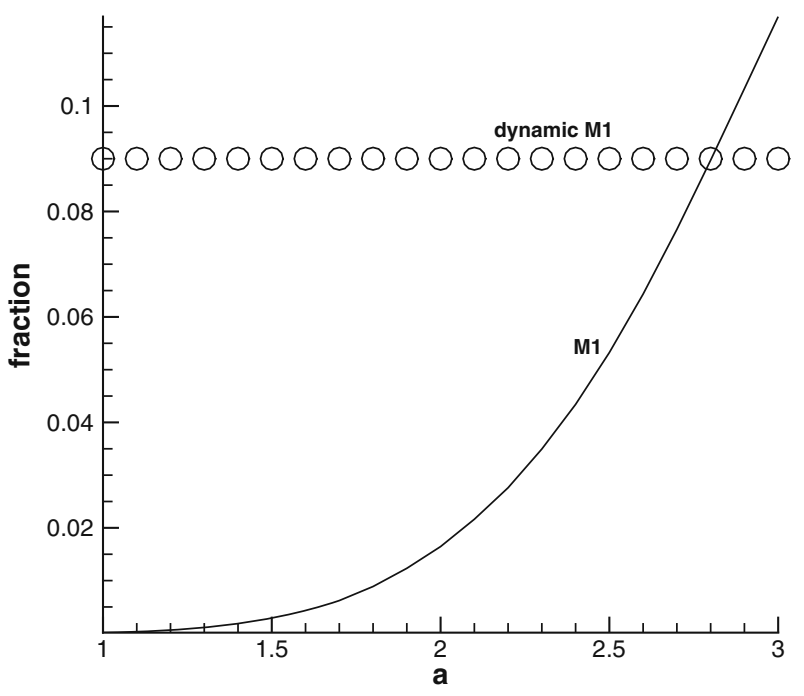




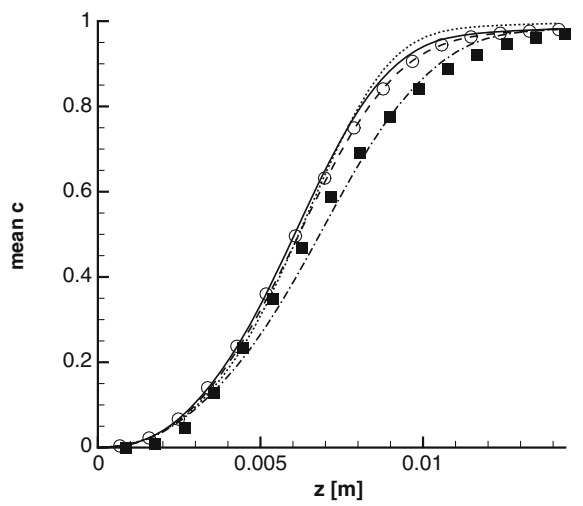

(a)

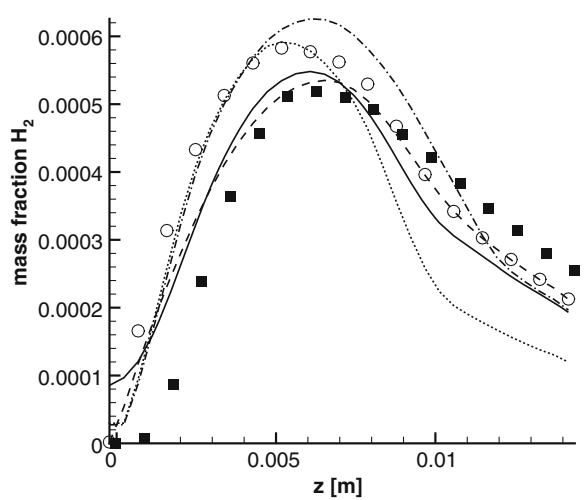

(c)

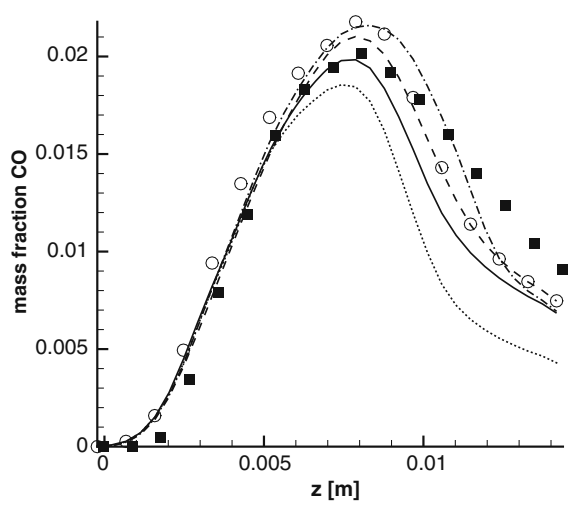

(b)

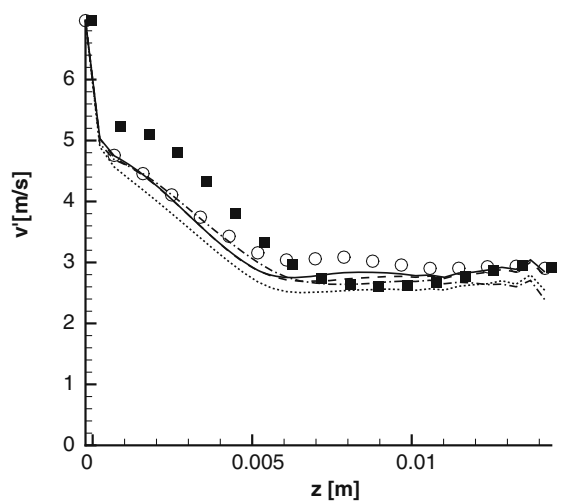

(d)

Fig. 7 Centerline mean progress variable (a), mean mass fractions of $\mathrm{CO}$ (b) and $\mathrm{H}_{2}$ (c), and turbulent intensity in the normal direction (d). Large-eddy simulation on $16 \times 16 \times 32$-grid with trivial model M0 (circles), with beta-pdf model M1 (solid), with filtered flamelet model M2 (dashed), with shift-filter model M3 (dotted), and with shift-inversion model M4 (dash-dotted); $a=2$ in case M1, M4 and M5. Results from DNS A are denoted with squares

the flame thickness approximately. Comparisons between LES and DNS for a case in which the LES does not resolve the flame thickness are presented in another paper [42].

For the subgrid reaction term, the models M0-4 are tested, with $a=2$ (where applicable). All large-eddy simulations used the eddy-viscosity (and eddy-diff usivity) that were specified in Section 2 (15). For the mean progress variable and mean fractions of most species there appears to be little difference between the different large-eddy simulations M0-3, but M4 makes a larger difference, and is closer to the DNS-result than the other large-eddy simulations. Unlike the other simulations, the simulations with M3 and M4 showed values for $c$ locally larger than 1 . This is clearly unphysical and a side effect of the (outer) filter used in these models. However, in practice the effect on the evolution of the flame may be small [42]. 
Figure $7 \mathrm{~b}$, c show the mass fractions of two species $\left(\mathrm{CO}\right.$ and $\left.\mathrm{H}_{2}\right)$ that show larger differences for the various models than the progress variable does. The deviations of $\mathrm{H}_{2}$ close to the entrance are explained by sensitivity to fluctuations of $c$; for low values of $c$ the mass fraction of hydrogen is very sensitive to a small change of $c$, due to the large preferential diffusion of hydrogen. For the species shown in Fig. 7b, c, the best results appear to be produced by the filtered flamelet model. Formally DNS should be filtered to compare fluctuating quantities (Fig. 7d), or the subgrid model or some sort of defiltering has to be used to retrieve the SGS part of the fluctuations. However, since we use a positive filter in our LES, we know that the subgrid parts of the diagonal components of the Reynolds stresses are positive [43]. This implies that LES intensities should be lower than their unfiltered DNS (or experimental) counterparts. Since the violation of this condition is more severe for M0 than for M1-4 (Fig. 7d); we can conclude that the models M1-4 produce better normal intensity than the trivial model M0. The overall impression given by Fig. 7 is that M2 is the best and M3 is the worst model, and this is consistent with the a priori predictions. Nevertheless, in view of its nice performance in the a priori tests, the behavior of M2 in the a posteriori tests is somewhat disappointing.

The overall impression is that M3 should not be used (at least not in the present form, M3 improves considerably if the explicit filter in the model is applied at a larger scale [42]), and that the other models do not much differ. Also the results of the latter models do not show large improvements over the trivial model M0. Apparently subgrid reaction effects are not very important when the filter width and flame thickness are of the same order. A much larger effect of subgrid reaction modeling has been observed for a Bunsen flame with flame thickness less than half the LES grid size [42].

The conclusions above should be viewed in the perspective that jet flows are usually sensitive to details of inflow conditions, turbulence modeling and numerics. Table 1 addresses the issue of numerics by comparing the $L_{2}$-norms of the a priori discretization errors in the scalar equations with the $L_{2}$-norms of the resolved and subgrid terms in the scalar equation. Since three terms (convective, viscous and source term) are nonlinear, three different subgrid terms are induced. The discretization errors listed in the table are those involved with spatial derivatives. They are calculated using the procedure described in Ref. [44]. The $L_{2}$ norms are calculated by spatial integration of the terms extracted from the DNS-field at a representative time. The discretization errors turn out to be relatively large. This means that the observed differences between LES and filtered DNS, which expresses the combined error of LES model and numerical scheme, were most probably influenced by the discretization error. This issue is further addressed in a posteriori tests in Ref. [42].

Table $1 L_{2}$-norms $\left[\mathrm{kg} \mathrm{m}^{-3 / 2} \mathrm{~s}^{-1}\right]$ of a priori LES-errors in the scalar equation $\left(\Delta=h_{L E S}=8 h_{D N S}\right)$, based on a snapshot of the DNS field

\begin{tabular}{llll}
\hline & Resolved part & Subgrid part & Discretization error \\
\hline Convective term & 1.98 & 0.10 & 1.35 \\
Viscous term & 0.18 & 0.05 & 0.29 \\
Source term & 0.78 & 0.39 & - \\
\hline
\end{tabular}




\section{Large-Eddy Simulation of a Partially Premixed Diffusion Flame (Sandia Flame D)}

In this section we present LES results for Sandia Flame D, a case for which experimental results are available. We performed LES using both premixed and non-premixed databases, the latter consisting out of steady non-premixed flamelets, where the non-covered part of the reaction regime was filled by linear interpolation.

The partially premixed Sandia Flame D is a piloted coaxial methane-air flame [12]. The fuel, a mixture of $25 \%$ methane and $75 \%$ air, corresponding to $Z=1$, leaves the inner nozzle (diameter $D=7.2 \mathrm{~mm}$ ) with a bulk velocity of $49.6 \mathrm{~m} / \mathrm{s}$. The pilot flow (bulk-velocity $10.8 \mathrm{~m} / \mathrm{s}$ and mixture fraction $Z=0.27$ ) exits from a wider nozzle, which is centered around the inner nozzle and has a diameter of $2.62 \mathrm{D}$. Around the outer nozzle there is a flow of air with a velocity of $0.9 \mathrm{~m} / \mathrm{s}$. The streamwise coordinate $z$ is defined to be zero at the nozzle exit. Experimental results were measured for $z<80 \mathrm{D}$. To reduce the effect of the outflow boundary conditions in the region of measurement, the computational domain was taken considerably longer; the numerical outflow conditions were imposed at $z=150 \mathrm{D}$. The extended length of the computational domain accounted for $20 \%$ of the number of grid-points. The computational domain was rectangular, such that a Cartesian grid could be used. A cross section of the flow domain was a horizontal square with a length of about $40 \mathrm{D}$ in each direction ( $x$ and $y$ ). The cells with centers $x=y=0$ were on the axis of the flow. The advantage of a Cartesian grid is that the explicit time-step can be larger than on cylindrical grids. The grid was stretched in all three directions and contained $128 \times 128 \times 320$ cells, with a minimum grid-spacing equal to $D / 5$ in the $z$-direction and $D / 8$ in the $x$ and $y$-directions. Figure 8 illustrates the influence of the Cartesian grid for a cylindrical flow. For a given downstream location, the mean streamwise velocity is shown for the four radial lines that align with the Cartesian grid. Also the velocity on the four radial lines rotated over an angle of $\pi / 4$ are shown. These

Fig. 8 Mean streamwise velocity at $z=35 D$ as a function of $r$ for the angles $\theta=0, \pi / 2, \pi, 3 \pi / 2$ (solid lines) and the angles $\theta=\pi / 4,3 \pi / 4,5 \pi / 4,7 \pi / 4$ (dashed lines)

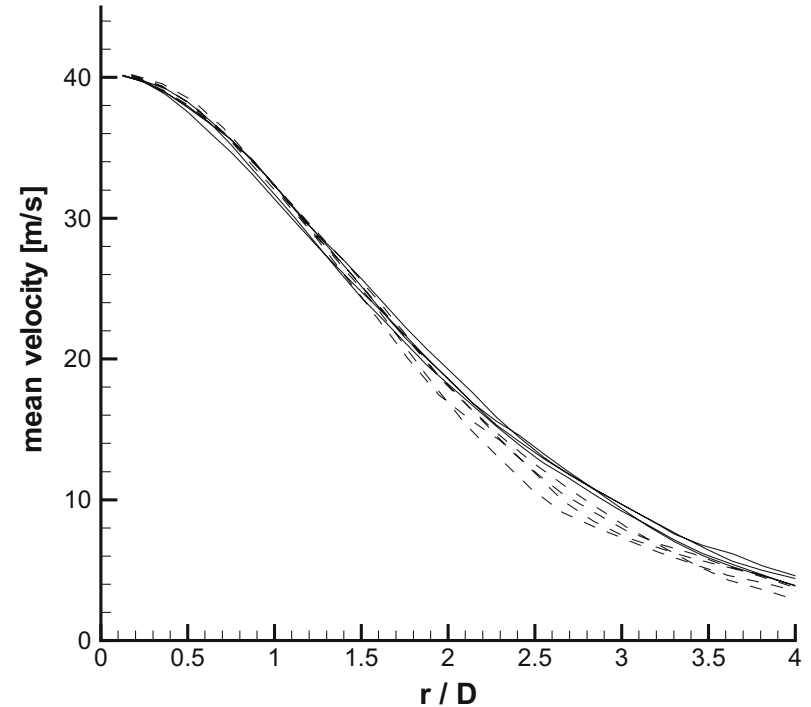


lines are not aligned with the Cartesian grid. The agreement between the curves is reasonable; differences are small for $r<1.5 \mathrm{D}$, giving confidence in the centerline statistics shown later on.

Outflow conditions in vertical and horizontal directions were zero normal derivatives for all variables, except for the pressure, which was maintained equal to the ambient pressure in outflow planes in the $x$ and $y$-directions. The simulation turned out to be sensitive to the inflow conditions, that is whether the experimental profiles measured at the nozzle or measured $7.2 \mathrm{~mm}$ downstream the nozzle were used as inflow conditions [21]. Here we used the latter profiles and perturbed the velocities with uniform noise, with amplitudes equal to the corresponding measured turbulent intensities.

The simulations were started from a state where all velocities and scalars were zero, except from the streamwise velocity $w$, which was initialized to the velocity of the outer flow $(0.9 \mathrm{~m} / \mathrm{s})$. The constant time step equalled $5 \cdot 10^{-6} \mathrm{~s}$. Time-averaging of statistics was started at $0.06 \mathrm{~s}$ (larger than the flow through time on the axis until $80 \mathrm{D}$ ), and all simulations reported in this and the following sections were run until approximately $0.16 \mathrm{~s}$. However, the premixed fine-grid simulation was continued beyond $0.3 \mathrm{~s}$, to verify that the statistics were converged at $0.16 \mathrm{~s}$.

\subsection{Results: LES with premixed and non-premixed flamelets}

In this subsection we compare the results of two Large-Eddy Simulations, one with the premixed database, the other with the non-premixed database. These simulations were both performed on the fine grid and for inlet conditions prescribed at $z=D$. Having verified that in this case the influence of beta-pdf modeling was small [21], we present only results in which the subgrid effect on quantities retrieved from the flamelet table is ignored. Flame D is not very far from equilibrium and the representative stretch of this flame is probably 400/s or less (the extinction border

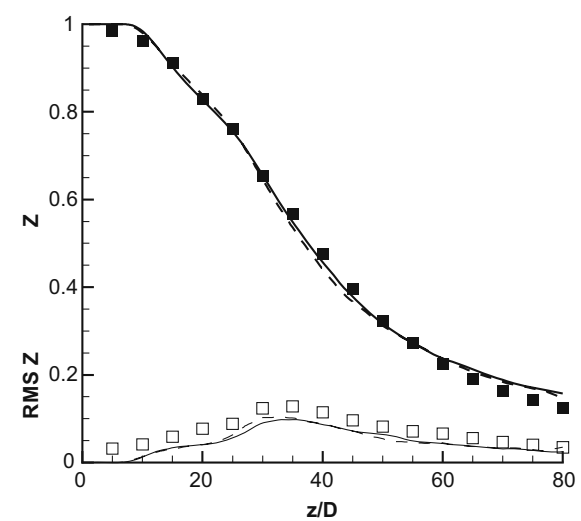

(a)

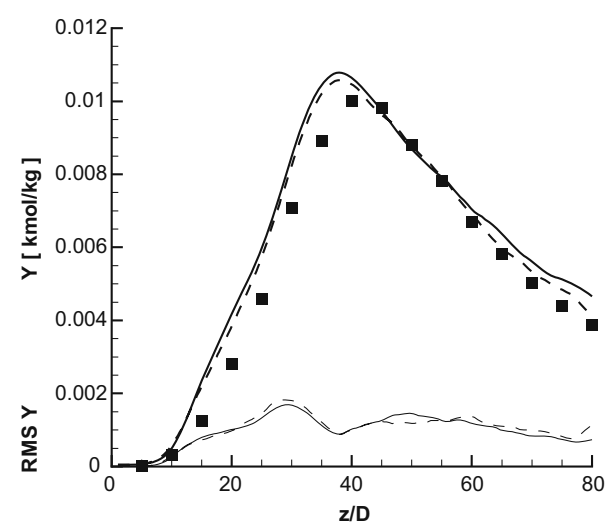

(b)

Fig. 9 Simulation results obtained with the premixed (solid lines) and the non-premixed flamelet database (dashed lines). Mean and fluctuation of mixture fraction (a) and progress variable (b) on the central axis. Thick lines represent mean values, thin lines the fluctuation. The experimental data from literature [12] is denoted with symbols 
for diffusion flamelet is about 1,200/s). Thus the non-premixed flame thickness, the square root of the quotient of the stoichiometric diff usion coefficient and stretch [38], is around $1.3 \mathrm{~mm}$. The smallest grid scale used in our simulations of flame D is smaller (present subsection) or of the same order (next subsection). One conclusion from the DNS-LES comparison in the previous section is that subgrid reaction effects are of minor importance in such a case.

We show statistical results for the mixture fraction and progress variable first. It is essential to obtain these quantities with accuracy, since in the present flamelet approach the entire chemistry depends on these two quantities. Figure 9 shows mean and fluctuation of the mixture fraction and progress variable on the centerline. Both the premixed and non-premixed case give a mean mixture fraction that is very close to the experimental values. The progress variable is somewhat overpredicted on the rich side of the flame. The overprediction is slightly larger for the premixed case,

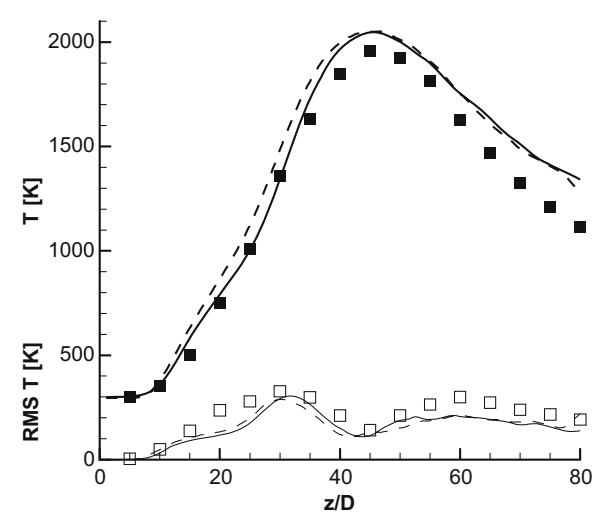

(a)

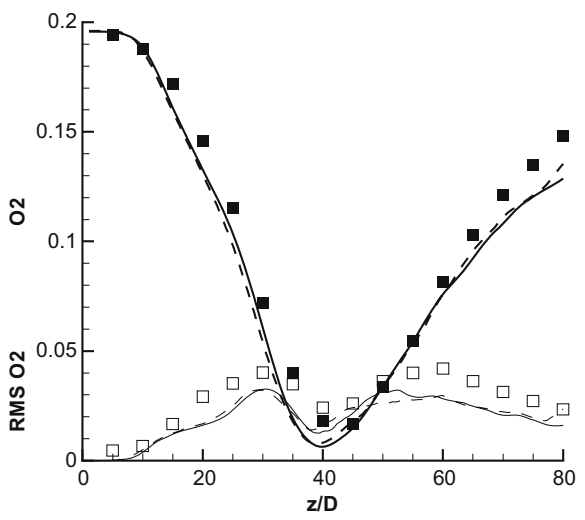

(b)

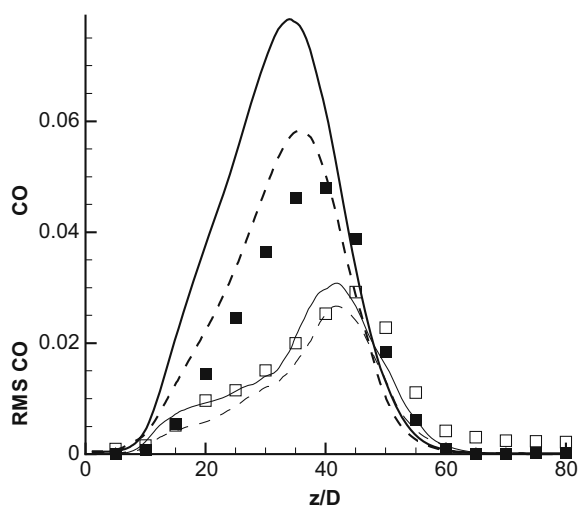

(c)

Fig. 10 Simulation results obtained with the premixed (solid lines) and the non-premixed flamelet database (dashed lines). Mean and fluctuation of temperature (a) and mass fractions of an example of a major species, $\mathrm{O}_{2}(\mathbf{b})$, and an example of a minor species, $\mathrm{CO}(\mathbf{c})$, on the central axis. Thick lines represent mean values, thin lines the fluctuation. The experimental data from literature [12] is denoted with symbols 
probably because the maximum of the source term of $Y$ in the premixed database appeared to be about $10 \%$ higher than in the non-premixed database [21]. The source term is not negligible, which was confirmed by a simulation without source term, showing significant differences. Because the source term matters, there is a significant deviation from chemical equilibrium in this flame, since in chemical equilibrium the source term would be zero.

Figure 10 shows the temperature, an example of a major species (the mass fraction of $\mathrm{O}_{2}$ ) and an example of a minor species (the mass fraction of $\mathrm{CO}$ ). Temperature and major species are well predicted by both premixed and non-premixed simulations. Larger deviations occur for the minor species. Here CO is over-predicted by both cases, but most by the premixed case. Inspection of the manifolds for rich values of the mixture fraction shows that also in the manifolds the premixed $\mathrm{CO}$ mass fraction is larger than the non-premixed one [21].

\subsection{Results: comparison between four eddy-viscosity models}

To assess the influence of the subgrid-model, we performed a simulation without a subgrid-model, and simulations for four eddy-viscosity models. All these simulations were performed on a coarser grid (1.5 times coarser in each direction).

The results are shown in Fig. 11. The mean mixture fraction appears to be very similar for three of the eddy-viscosity models: the model given by equation (15), the dynamic Smagorinsky model, and the WALE model. Although these simulations were performed on a coarser mesh, the results for the mean mixture fraction are hardly different from those on the fine mesh (compare Fig. 9).

However the mean mixture fraction in the cases without subgrid model and with the standard Smagorinsky model $\left(C_{S}=0.1\right)$ is quite different from the other three

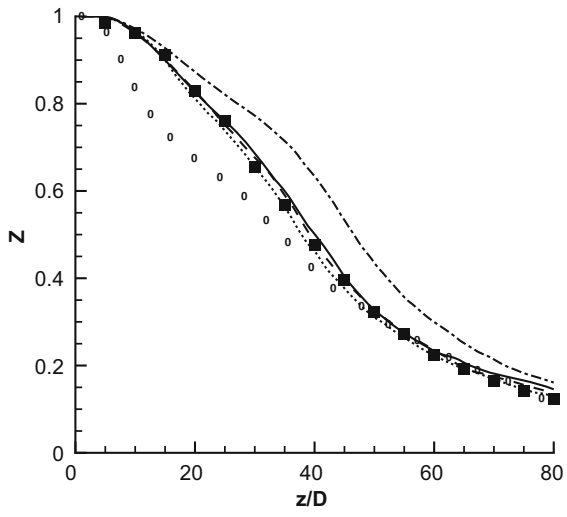

(a)

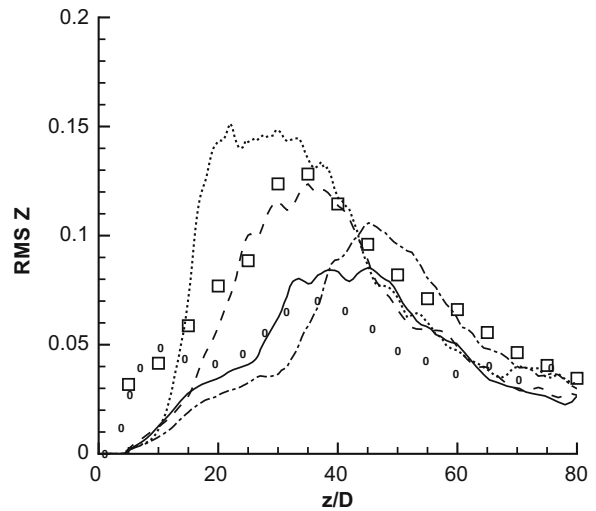

(b)

Fig. 11 Mean (a) and rms (b) mixture fraction at the centerline for different eddy-viscosity subgrid models. Simulation without subgrid model (symbol ' 0 ') and LES with model proposed by Vreman [31], dynamic Smagorinsky model [34] (dashed), WALE model [35] (dotted), and standard Smagorinsky model (dash-dotted). The experimental data from literature [12] is denoted with squares. Simulation results have been obtained with the premixed flamelet database, on the coarser grid $(80 \times 80 \times 192)$ 
models and also from the experimental results. The strong discrepancies between the simulation without subgrid model and simulations with subgrid models indicate that the three best subgrid models improve the simulations indeed.

Considering the fluctuation of mixture fraction (lower part of the figure), the dynamic curve is closest to the experimental result. However, this does not necessarily mean that results of the dynamic model are the best, since in principle the LES fluctuations should be smaller than the experimental ones. The dynamic model does not satisfy this requirement at the rich side of the flame, where turbulence is relatively important. At the same location $(z / D \approx 25)$, the fluctuation of the WALE model is even larger than the experimental values. These relatively large fluctuations may be related to the fact that the dynamic and WALE eddy-viscosities are less smooth than the other two eddy-viscosities.

\section{Conclusions}

Large-eddy simulation with premixed flamelet chemistry was studied by comparison with DNS and comparison with experimental data. An efficient low-Mach numerical scheme was used. In the first case a premixed preheated planar Bunsen flame was simulated. Both LES and DNS were performed with flamelet chemistry. The DNS was validated to be grid independent, and the turbulent flame was verified to be in the thin reaction zone regime, with $u^{\prime} / s_{L 0}$ about 3 and the Karlovitz number about 9 (based on maximum temperature gradient flame thickness and Kolmogorov length). The turbulent burning velocity was about $2.1 s_{L 0}$. This DNS was used to test three subgrid modeling approaches for the chemical terms. The standard beta-pdf approach suffers from the fact that the subgrid scalar variance is unknown. The model for this term reduces the accuracy of the approach considerably. Therefore we considered three simpler approaches: the filtered flamelet, the shift-filter and the shift-inversion model. The filtered flamelet model, in which the LES uses a spatially prefiltered flamelet, appears to be more accurate than the other models, including the beta-pdf approach, at least in a priori tests. However, in a posteriori tests and considering a range of profiles, no single model appeared to be better than the other models. The conclusion is that, in case the flame thickness is of the same order as the filter width (grid spacing), subgrid reaction modeling is not important, or less important than the discretization error. If the flame thickness is smaller than half the grid size, a posteriori results show much more sensitivity to subgrid reaction modeling, see Ref. [42], where also the role of the discretization error has been considered in more detail. For the second case, the comparison with experimental data, we performed LES of a partially premixed jet diff usion flame, Sandia Flame D. Several subgrid models were compared, the Smagorinsky eddy-viscosity, where the mean shear can be quite dominant, and three other models with less dependence on mean shear. The latter three models produced results closer to the experimental data than the Smagorinsky model. For the generality of our approach it was interesting to learn that the premixed LES strategy works reasonably well, also for partially premixed flames.

Acknowledgement This research was funded by Stichting Technische Wetenschappen, grant number EWO.5874. 
Open Access This article is distributed under the terms of the Creative Commons Attribution Noncommercial License which permits any noncommercial use, distribution, and reproduction in any medium, provided the original author(s) and source are credited.

\section{References}

1. Bilger, R.W., Pope, S.B., Bray, K.N.C., Driscoll, J.F.: Paradigms in turbulent combustion research. Proc. Comb. Inst. 30, 21-42 (2005)

2. Pitsch, H.: Large-eddy simulation of turbulent combustion. Annu. Rev. Fluid Mech. 38, 453-482 (2006)

3. Peters, N.: Laminar diffusion flamelet models in non-premixed turbulent combustion. Prog. Energy Combust. Sci. 10, 319-339 (1984)

4. Pierce, C.D., Moin, P.: Progress-variable approach for large-eddy simulation of non-premixed turbulent combustion. J. Fluid Mech. 504, 73-97 (2004)

5. Van Oijen, J.A.: Flamelet-generated manifolds: development and application to premixed laminar flames. PhD. Thesis, TU Eindhoven (2002)

6. Bradley, D., Kwa, L.K., Lau, A.K.C., Missaghi, M., Chin, S.B.: Laminar flamelet modeling of recirculating premixed methane and propane-air combustion. Comb. Flame 71, 109-122 (1988)

7. Van Oijen, J.A., De Goey, L.P.H.: Modelling of premixed laminar flames using flameletgenerated manifolds. Combust. Sci. and Tech. 161, 113-137 (2000)

8. Gicquel, O., Darabiha, N., Thev́enin, D.: Laminar premixed hydrogen/air counterflow flame simulations using flame prolongation of ILDM with differential diffustion. Proc. Combust. Inst. 28, 1901-1908 (2000)

9. Filatyev, S.A., Driscoll, J.F., Carter, C.D., Donbar, J.M.: Measured properties of turbulent premixed flames for model assessment, including burning velocities, stretch rates, and surface densities. Comb. Flame 141, 1-21 (2005)

10. Bell, J.B., Day, M.S., Grcar, J.F., Lijewski, M.J., Driscoll, J.F., Filatyev, S.A.: Numerical simulation of a laboratory-scale turbulent slot flame. Proc. Comb. Inst. 31, 1299-1307 (2007)

11. Sankaran, R., Hawkes, E.R., Chen, J.H., Lu, T., Law, C.K.: Structure of a spatially developing turbulent lean methane-air Bunsen flame. Proc. Comb. Inst. 31, 1291-1298 (2007)

12. Barlow, R.S., Frank, J.H.: Effects of turbulence on species mass fractions in methane/ air jet flames. Proc. Combust. Inst. 27, 1087-1095 (1998). Experimental database at www.ca. sandia.gov/TNF/DataArch/FlameD.html

13. Schneider, C., Dreizler, A., Janicka, J., Hassel, E.: Flow field measurements of stable and locally extinguishing hydrocarbon-fuelled jet flames. Comb. Flame 135, 185-190 (2003)

14. Pitsch, H., Steiner, H.: Large-eddy simulation of a turbulent piloted methane/air diffusion flame (Sandia flame D). Phys. Fluids 12, 2541-2554 (2000)

15. Kempf, A.M.: Large-eddy simulation of non-premixed turbulent flames. PhD-Thesis, TU Darmstadt (2003)

16. Goldin, G.M.: Evaluation of LES subgrid reaction models in a lifted flame. AIAA Paper 2005$555,1-13(2005)$

17. Geiss, C.: Investigation of kinetic effects in turbulent non-premixed flames by means of LES and FGM. Diplomarbeit, TU Darmstadt (2005)

18. Sheikhi, M.R.H., Drozda, T.G., Givi, P., Jaberi, F.A., Pope, S.B.: Large eddy simulation of a turbulent nonpremixed piloted methane jet flame (Sandia Flame D). Proc. Comb. Inst. 30, 549-556 (2005)

19. Mustata, R., Valino, L., Jimenez, C., Jones, W.P., Bondi, S.: A probability density function Eulerian Monte Carlo field method for large eddy simulations: application to a turbulent piloted methane/air diffusion flame (Sandia D). Comb. Flame 145, 88-104 (2006)

20. Van der Hoeven, S., Boersma, B.J., Jonker, H.J.J., Roekaerts, D.J.E.M.: Development of a large eddy simulation code for turbulent non-premixed jet flames. Proceedings Computational Combustion (2007)

21. Vreman, A.W., Albrecht, B.A., van Oijen, J.A., de Goey, L.P.H., Bastiaans, R.J.M.: Premixed and non-premixed generated manifolds in large-eddy simulation of Sandia flame D and F. Comb. Flame 153(3), 394-416 (2008)

22. Bradley, D., Emerson, D.R., Gaskell, P.H., Gu, X.J.: Mathematical modeling of turbulent nonpremixed piloted-jet flames with local extinctions. Proc. Combust. Inst. 29, 2155-2162 (2002) 
23. Merci, B., Dick, E., Vierendeels, J., de Langhe, C.: Determination of $\epsilon$ at inlet boundaries. Int. J. Heat Fluid Flow 12, 65-80 (2002)

24. Cao, R.R., Pope, S.B.: The influence of chemical mechanisms on PDF calculation of nonpremixed piloted jet flames. Comb. Flame 143, 450-470 (2005)

25. Cao, R.R., Wang, H., Pope, S.B.: The effect of mixing models in PDF calculations of piloted jet flames. Proc. Comb. Inst. 31, 1543-1550 (2007)

26. Ramaekers, W.J.S.: The application of flamelet generated manifolds in modelling of turbulent partially-premixed flames. Master thesis, TU Eindhoven (2005)

27. Albrecht, B.A., Ramaekers, W.J.S., van Oijen, J.A., Bastiaans, R.J.M., de Goey, L.P.H.: The application of flamelet generated manifolds in modeling of turbulent partially premixed flames, preprint (2007)

28. Fiorina, B., Gicquel, O., Vervisch, L., Carpentier, S., Darabiha, N.: Approximating the chemical structure of partially premixed and diffusion counterflow flames using FPI flamelet tabulation. Comb. Flame 140, 147-160 (2005)

29. Bongers, H., van Oijen, J.A., Somers, L.M.T., de Goey, L.P.H.: The flamelet generated manifold method applied to steady planar partially premixed counterflow flames. Combust. Sci. and Tech. 177, 2373-2393 (2005)

30. Smooke, M.D., Giovangigli, V.: Formulation of the premixed and nonpremixed test problems. In: Smooke, M.D. (ed.) Reduced Kinetic Mechanisms and Asymptotic Approximations for Methane-air Flames, pp. 1-28. Springer Verlag Berlin (1991)

31. Vreman, A.W.: An eddy-viscosity model for turbulent shear-flow: algebraic theory and applications. Phys. Fluids 16, 3670-3681 (2004)

32. Leonard, A.: Energy cascade in large-eddy simulations of turbulent fluid flows. Adv. Geophys. 18, 237 (1974)

33. Clark, R.A., Ferziger, J.H., Reynolds, W.C.: Evaluation of subgrid-scale models using an accurately simulated turbulent flow. J. Fluid Mech. 91, 1-16 (1979)

34. Germano, M., Piomelli, U., Moin, P., Cabot, W.: A dynamic subgrid-scale model. Phys. Fluids A 3, 1760-1765 (1991)

35. Nicoud, F., Ducros, F.: Subgrid-scale stress modelling based on the square of the velocity gradient tensor. Flow Turbul. Combust. 62, 183-200 (1999)

36. van der Burg, J.W.: Numerical techniques for transonic flow calculations. PhD Thesis, University of Twente (1993)

37. Wesseling, P.: An Introduction to Multigrid Methods. Wiley (1992)

38. Peters, N.: Turbulent Combustion. Cambridge University Press (2000)

39. Cook, A.W., Riley, J.J.: A subgrid model for equilibrium chemistry in turbulent flows. Phys. Fluids 6, 2868-2870 (1994)

40. Domingo, P., Vervisch, L., Payet, S., Hauguel, R.: DNS of a premixed turbulent V flame and LES of a ducted flame using a FSD-PDF subgrid scale closure with FPI-tabulated chemistry. Comb. Flame 143, 566-586 (2005)

41. Geurts, B.J.: Regularization modeling for large-eddy simulation of diffusion flames. Proceedings ECCOMAS CFD 2006 (2006)

42. Vreman, A.W., Bastiaans, R.J.M., Geurts, B.J.: Accuracy of large-eddy simulation of premixed turbulent combustion. Submitted to Flow Turbul. Combust. (2008)

43. Vreman, B., Geurts, B., Kuerten, H.: Realizability conditions for the turbulent stress tensor in large eddy simulation. J. Fluid Mech. 278, 351-364 (1994)

44. Vreman, B., Geurts, B., Kuerten, H.: A priori tests of large-eddy simulation of the compressible plane mixing layer. J. Engg. Math. 29, 299-327 (2005) 